\title{
Evolución de los sistemas de gestión de contenidos (CMS). Del mainframe al open source
}

\author{
Por Jorge Serrano-Cobos
}

\begin{abstract}
Resumen: Los sistemas de gestión de contenidos o content management systems (CMS) tienen por misión automatizar la generación, mantenimiento y recuperación de información digital, permitiendo llevar el contenido correcto a la persona adecuada en su momento justo al coste idóneo. En el artículo se estudia el concepto de CMS y su evolución desde los años 70 hasta las herramientas de código abierto (open source) en boga a partir de 2000, así como su relación con otros sistemas como los de gestión documental (SGD), records management o ECM (enterprise content management). Finalmente se reseñan las funcionalidades propias de un CMS, y se ofrecen unos consejos prácticos para elegir un software apropiado a las necesidades de cada servicio de información.

Palabras clave: Sistemas de gestión de contenidos, Sistemas de información, Gestores de contenidos, Content management systems, CMS, Web content management systems, WCMS, ECM, Enterprise content management.
\end{abstract}

Title: Evolution of content management systems (CMS). From mainframe to open source

Abstract: Content management systems (CMS) let us automate the generation, management, and retrieval of digital information, giving us the ability to deliver the right content to the right people at the right time at the right cost. This article analyzes the concept of CMS, its evolution from the 1970s to the open source tools developed since 2000, and its relationship to other systems, such as document management, records management or enterprise content management. Finally, a set of basic functions is detailed, along with practical advice

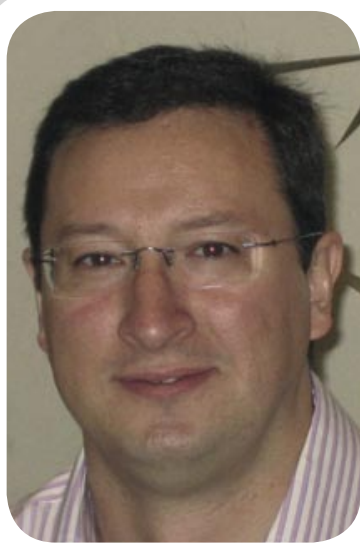

Jorge Serrano-Cobos es diplomado en ByD por la Univ. de Zaragoza, donde actualmente cursa doctorado. Ldo. en Documentación por la Univ. de Granada. Postgrado en Comunicación en Lenguaje Multimedia por la Univ. de Zaragoza. Jefe del Depto. de Contenidos de MASmedios. to help determine the best CMS to meet the specific needs of any particular information service.

Keywords: Information systems, Content management systems, CMS, Web content management systems, WCMS, Enterprise content management, ECM.

Serrano-Cobos, Jorge. "Evolución de los sistemas de gestión de contenidos (CMS). Del mainframe al open source". En: El profesional de la información, 2007, mayo-junio, v. 16, n. 3, pp. 213-215.

DOI: 10.3145/epi.2007.may.05

ES POSIBLE QUE COMO PROFESIONALES de la información un día maquetásemos una página web con los pocos conocimientos de html que teníamos, y ahora se nos haya quedado pequeña, pues necesitamos incluir al menos noticias e imágenes, y crear las páginas desde cero una a una resulta una tarea muy ardua.

Puede que debamos pergeñar un sistema de información global para un hospital, en el que se integren radiografías digitales, electrocardiogramas, ecografías en 2,3 y 4D, archivos de audio de latidos del corazón, la evolución de la posología hora a hora y todos los datos imaginables de las enfermedades asociadas a cada historial clínico de nuestros pacientes, para que cada médico, enfermera y especialista pueda por un lado incluir esos datos mediante diversos métodos en el repositorio, y otro usuario del sistema lo pueda consultar instantáneamente en cualquier dispositivo preparado a tal efecto.

Acaso nos hayan encargado rediseñar el portal corporativo de nuestra empresa o institución, e incluir información actualizada de todos los planos, maquetas, proyectos, noticias, relaciones con la administración, facturas u ofertas de empleo que la empresa genera a diario, integrando toda la información en cualquier formato y soporte en un todo organizado consultable a través de una intranet y de otros dispositivos como los móviles, por ejemplo.

Incluso quizá, se haya decidido en la biblioteca que se debería incorporar el opac a un portal bibliotecario en el que los usuarios puedan conocer algo más que el catálogo de la misma, permitirles visionar vídeos o escuchar grabaciones radiofónicas desde su casa, e interactuar entre ellos incorporando sus propias reseñas, comentarios, generando comunidades de lectores aficionados a tal o cual género literario, a través de un flujo de trabajo en el que el bibliotecario pueda permitir la publicación o no de determinados contenidos ofrecidos por otros usuarios. 
Probablemente si alguien se halla en esa tesitura, puede necesitar un $C M S$.

\section{Para qué un CMS}

¿De qué estamos hablando?, ¿cómo nos puede ayudar a desarrollar nuestro trabajo, y cuál debemos elegir?

Se estima que existen en la actualidad unos 2.000-3.000 sistemas de gestión de contenidos, o content management systems (CMS) en todo el mundo ${ }^{1}$. Básicamente, tienen tres funciones vitales: permitir la entrada de contenido digital de cualquier clase, permitir su gestión, y por supuesto en última instancia, su recuperación futura. En palabras de Gerry Macgovern, "la gestión de contenidos consiste en llevar el contenido correcto a la persona adecuada en su momento justo al coste idóneo"2.

\section{Breve historia}

El concepto de CMS ha experimentado una fuerte evolución, y no es lo mismo una aplicación de esta naturaleza de hace 10 años que ahora; ni se le pedía lo mismo. Históricamente nacen en los años 70 en el entorno de los grandes ordenadores o mainframes. No sería hasta el año 1984 cuando se conocen los primeros sistemas que permiten publicar contenido desde un ordenador personal, y sólo desde 1995 podemos hablar de gestión de contenido en y para internet, lo que se ha dado en denominar WCMS (web content management systems) ${ }^{3}$.

Debido a su evolución, maduración natural del mercado y su reciente popularización gracias a las herramientas open source y una necesidad de simplificación del mensaje por parte de los proveedores -branding (imagen de marca) obliga-, el concepto de CMS ha ido ganando más y más adeptos, incluyendo o canibalizando a otros que definían herramientas más específicas con las que existía acaso cierto solapamiento, como los sistemas de publicación de información personal (lo que eran los blogs en su día) los $S G D$ (sistemas de gestión documental, especializados en su momento en gestionar documentos, que no información en sí misma, independientemente de su soporte) records management y ECM (enterprise content management) entre otras mil siglas diferentes ${ }^{4}$.

\section{Funciones propias de un CMS}

Más que definirlo, del que cabría generar toda una clasificación (que no taxonomía mutuamente excluyente, debido a las zonas grises entre clases de software) con diversas familias, géneros y subgéneros, sí podríamos apuntar algunas características genéricas que cumpliría en mayor o menor medida una herramienta que quiera ser denominada como tal ${ }^{5}$ :

- Un repositorio de datos común.

- Separación del contenido de la presentación del mismo.

- Edición wysiwyg, con editores de texto tipo Word o similar.

- Flujo de trabajo o workflow en la edición o aprobación de la publicación de contenidos, junto a la automatización de tareas.

- Check in y check out (regla por la que un usuario no puede edi- tar el texto que estoy escribiendo hasta que no lo haya guardado yo).

- Control de seguridad y de usuarios.

- Control de versiones, de forma que se pueda ver quién ha modificado qué y cuándo.

- Gestión de metadatos, lo que incluye clasificación del contenido.

- Reutilización del contenido una vez introducido.

- Integración de diversos formatos de contenido (texto, sonido, imagen, video, etc.).

- Interoperabilidad multicanal para poder generar versiones impresas, en pdf, PDAs, móviles, etc., desde un formato inicial de entrada.

- Personalización ("mi portal").

- Traducción multilingüe.

Entre otras más. Y cada día se añaden nuevas capacidades. A partir de ahí, cada proveedor incorporará más y mejores cualidades, unos especializándose en unas características, otros permitiendo realizar cualquier tipo de personalización de la aplicación que sirva a los intereses del usuario del sistema. Por lo general se podrá pensar que, cuanto más abierto sea (más posibilidades de ser modificado) más compleja será su implementación. Todo dependerá de nuestra imaginación y necesidades, como diseñadores y analistas funcionales del sistema.

\section{EI mejor CMS}

Así pues, ¿cuál de las ofertas del mercado será la que voy a necesitar? No hay una respuesta fácil, ni se puede (ni se debe) ofrecer un único software como el más adecuado para cualquier proyecto, por lo que sí podremos apuntar algunos consejos prácticos, unas heurísticas básicas:

- No pienses en un software para todo, piensa en uno para un proyecto. 
- Analiza primero el proyecto. Funciones, requerimientos, etc.

- Determina la posible evolución futura del proyecto (más necesidades).

- Genera prototipos del siste$\mathrm{ma} /$ proyecto aunque sea en papel, workflows, etc. Suele ser lo último que se hace antes de elegir software y debería ser de las primeras tareas a realizar. Te ayudará a delimitar lo que realmente necesitas, desechando lo que sobra, o ampliando lo que imaginabas teóricamente.

- Testea esos prototipos con usuarios probables del sistema. Te darán pistas para volver al punto anterior.

- ¿Cuánto presupuesto tienes?

- ¿Tienes equipo propio preparado para la gestión informática del proyecto a medio-largo plazo tras la implantación, o deberás contar con ayuda externa? ¿qué costes se incluyen en cada caso?

- Por último: quién está detrás de ese software: qué empresa, qué

\section{"No se puede (ni se debe) ofrecer un único software como el más adecuado para cualquier proyecto"}

equipo, qué comunidad de desarrolladores, qué experiencias anteriores, qué lo avala para tu proyecto.

- Vuelve al primer punto.

Con estos sencillos pasos estarás equipado para desbrozar la maraña del bosque de gestores de contenidos que se abrirá a nuestro alcance: unos más sencillos, otros más complejos, aquellos más abiertos, éstos más cerrados y verticales pero más rápidos de implantar; unos de software propietario, más o menos contrastados, otros open source, baratos (que no gratis porque alguien deberá dedicar horas a su implementación, y siempre hay una curva de aprendizaje) pero en constante evolución y en principio sin un soporte profesional detrás.

En nuestra búsqueda encontraremos multitud de listados ${ }^{6}$ ran-

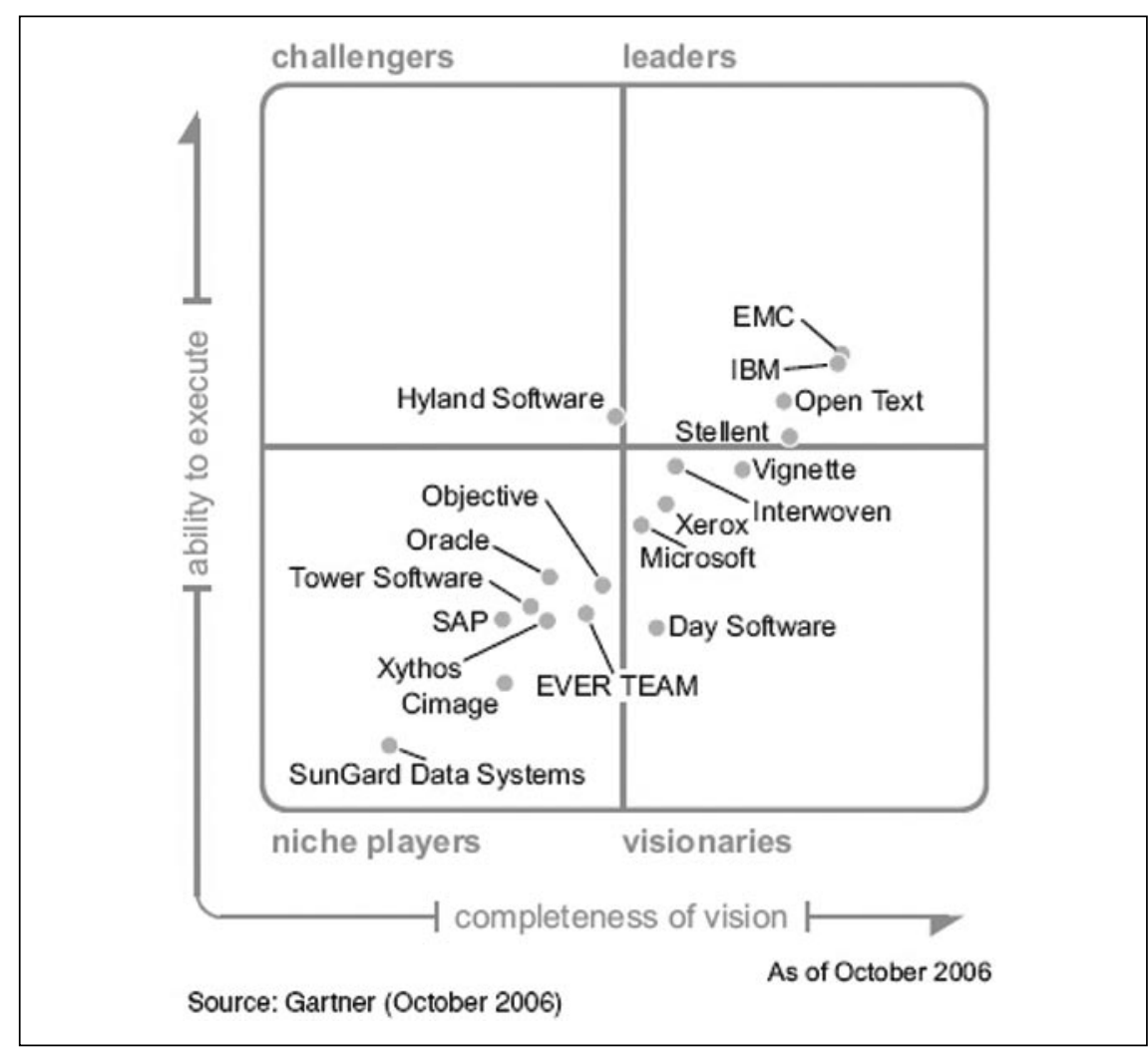

Posicionamiento en el mercado mundial de CMS de pago (Stellent, 2006) kings, cuadrantes mágicos o magic quadrants ${ }^{7}$ posicionando gráficamente a las empresas contendientes en el conjunto del mercado mundial de $C M S$, generados por grandes consultoras como Gartner ${ }^{8}$, y comparativas online'; todo es investigar sus características y cruzarlas con mis necesidades. Puede que no necesitemos matar moscas a cañonazos, y puede que necesitemos un obús. Depende.

Por tanto, la cuestión final será, invariablemente, no cuál es el mejor $C M S$ del mundo, sino cuál es mejor para nosotros.

\section{Notas bibliográficas}

1. CMSwatch. Consultado en: 21-03-07. http://www.cmswatch.com/

2. CMSCalendar. History of CMS. Consultado en: 21-03-07.

http://www.cmscalendar.com/glossary. html?term=HistoryOfCMS

3. Doyle, Bob. CMS genesis: who did what when, 2004. Consultado en: 21-03-07.

http://www.econtentmag.com/Articles/ArticleReader.aspx?ArticleID $=6819$

4. Contentmanager. Consultado en: 21-03-07. http://www.contentmanager.eu.com/history.htm

5. CMSReview. CMS review feature list. Consultado en: 21-03-07.

http://www.cmsreview.com/Features/Lists.html

6. Wikipedia. List of content management systems. Consultado en: 21-03-07.

http://en.wikipedia.org/wiki/List_of_content_ management_systems

7. Gartner. Magic quadrants and marketscopes. Consultado en: 21-03-07.

http://www.gartner.com/it/products/mq/mq_ ms.jsp

8. Stellent. Magic quadrant for enterprise content management, 2006. Consultado en: 21-03-07. http://www.stellent.com/stellent3/groups/mkt/ documents/nativepage/gartnerecmmq.pdf

9. CMSmatrix. The content management comparison tool. Consultado en: 21-03-07. http://www.cmsmatrix.org/

Jorge Serrano-Cobos, MASmedios. http://www.masmedios.com/ jorgeserrano@gmail.com 